\title{
Two Novel Mutations and a previously unreported intronic polymorphism in the NOTCH3 gene
}

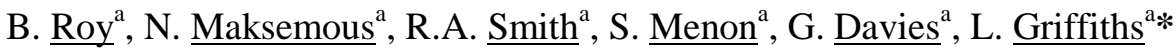 \\ ${ }^{a}$ Genomics Research Centre, Griffith Health Institute, Building G05, Griffith University Gold Coast, \\ Parklands Drive, Southport, Queensland, Australia, 4222 \\ *Author for correspondence \\ E-mail: 1.griffiths@griffith.edu.au \\ Phone: +61 (0)7 55528664 \\ Fax: +61(0)7 55529081
}

\begin{abstract}
Cerebral autosomal dominant arteriopathy with subcortical infarcts and leukoencephalopathy (CADASIL) is a hereditary disease of small vessel caused by mutations in the NOTCH3 gene (NCBI Gene ID: 4854) located on chromosome 19p13.1. NOTCH3 consists of 33 exons which encode a protein of 2321 amino acids. Exons 3 and 4 were found to be mutation hotspots, containing more than $65 \%$ of all CADASIL mutations. We performed direct sequencing on an ABI 3130 Genetic Analyser to screen for mutations and polymorphisms on 300 patients who were clinically suspected to have CADASIL. First, exons 3 and 4 were screened in NOTCH3 and if there were no variations found, then extended CADASIL testing (exons 2, 11, 18 and 19) was offered to patients. Here we report two novel non-synonymous mutations identified in the NOTCH3 gene. The first mutation, located in exon 4 was found in a 49 year-old female and causes an alanine to valine amino acid change at position 202 (590C $>$ T). The second mutation, located in exon 11, was found in a 66 year-old female and causes a cysteine to arginine amino acid change at position 579 (1735T >C). We also report a 46 year-old male with a known polymorphism Thr101Thr (rs3815188) and an unreported polymorphism NM_000435.2:c.680+60A>G observed in intron 4 of the NOTCH3 gene. Although Ala202Ala (rs1043994) is a common polymorphism in the NOTCH3 gene, our reported novel mutation (Ala202Val) causes an amino acid change at the same locus. Our other reported mutation (Cys579Arg) correlates well with other known mutations in NOTCH3, as the majority of the CADASIL-associated mutations in NOTCH3 generally occur in the EGF-like (epidermal growth factorlike) repeat domain, causing a change in the number of cysteine residues. The intronic polymorphism NM_000435.2:c.680+60A>G lies close to the intron-exon boundary and may affect the splicing mechanism in the NOTCH3 gene.
\end{abstract}

\begin{abstract}
Abbreviations: NOTCH3 (Notch homolog 3 (Drosophila)); CADASIL (Cerebral autosomal dominant arteriopathy with subcortical infarcts and leukoencephalopathy); EGF (epidermal growth factor); MRI (magnetic resonance imaging); GOM (granular osmiophilic material)
\end{abstract}

Keywords: NOTCH3; CADASIL; novel mutations; polymorphism 


\section{Highlights:}

$>$ CADASIL is a hereditary disease of small vessel caused by mutations in NOTCH3

$>$ Exons 3 and 4 were found to be mutation hotspots

$>$ We screened 300 patients who were clinically suspected to have CADASIL

$>$ We report two novel mutations in NOTCH3: Ala202Val (exon 4) and Cys579Arg (exon11)

$>$ We also report a polymorphism NM_000435.2:c.680+60A>G in intron 4

\section{Introduction}

Sourander and Walinder [1,2] described a cerebral vascular disease in 1977 which was previously called hereditary multi-infarct dementia. In 1993, Tournier-Lasserve coined the term CADASIL for the disorder [3]. CADASIL is a systemic vascular disorder that is clinically restricted to the central nervous system. The primary characteristic features of CADASIL are migraine, recurrent subcortical strokes, depression and vascular dementia, also associated with diffuse leukoencephalopathy on brain imaging [4]. The two main neuroimaging features of CADASIL on magnetic resonance imaging (MRI) are multiple subcortical infarcts and white matter hyperintensities [5]. At the ultrastructural level, the trademark of CADASIL is the occurrence of granular osmiophilic material (GOM) without filament-like profiles [6] adjoining smooth muscle cells in the vessel wall and generally located in smooth muscle cell infoldings. The nature of GOM still remains unclear [4].

CADASIL was mapped to the NOTCH3 gene on chromosome 19p13.1 [7] and mutations in this gene have been recognized as the genetic cause of CADASIL [8]. This gene has 33 exons that encode the Notch3 protein which consists of 2321 amino acids and is part of the Notch family involved in signaling activities that control cell fate decisions during development $[9,10]$. It is also a single-pass transmembrane receptor with a large extracellular domain enclosing 34 tandem epidermal growth factorlike (EGF-like) repeats. Notch3 is expressed in vascular smooth muscle cells in adult human tissues [4]. Proteolytic fragmentation of the Notch3 protein results in a small transmembrane domain of $97 \mathrm{kDa}$ and a large extracellular ectodomain of $210 \mathrm{kDa}$. It was found that in CADASIL, the $210 \mathrm{kDa}$ fragment builds up inside vessel walls that are near GOMs and this can be visualized by immunohistochemical methods [11]. Notch3 protein also undergoes three proteolytic cleavages during maturation [4]. This disorder was generally considered as a familial disease with an autosomal dominant pattern of inheritance [2]. In 2000, a study illustrated that CADASIL may be more frequent than initially expected and from the year 1993 up to 2000, more than 400 families were recognized, thus suggesting that diagnosis should not be limited in the absence of a familial history [12]. In 1997, Joutel and group [13] reported that the pathogenetic mutations in CADASIL patients were found to occur in strong clusters in exons 3 and 4 which encode the first five EGF repeats and were stereotyped in nature. This made the detection of these mutations easy and a reliable diagnostic tool for CADASIL. Most CADASIL mutations lead to an odd number of cysteines in the EGF-like repeats of the Notch3 protein. This group also suggested that the ideal genetic screening approach should 
consist of two steps that would be successful in $90 \%$ of patients. The first step was to sequence exons 3 and 4 to screen for mutations and if the result was negative for these two exons, then the other 22 implicated exons should be screened to identify mutations in patients showing clinical and MRI features consistent with CADASIL diagnosis.

Unfortunately, there is no treatment currently available precisely for CADASIL, although a recent study in 2010 showed that acetazolamide (ACZ) may be a potential candidate for CADASIL prophylactic treatment [14]. In the present study, we performed molecular testing of 300 patients clinically suspected with CADASIL. We first performed testing for exons 3 and 4 in the NOTCH3 gene as these regions are most commonly known to harbour mutations and if these exons showed no variations, extended CADASIL testing (exons 2, 11, 18 and 19 in the NOTCH3 gene) was offered to patients. We observed thirteen different mutations in patients, of which Ala202Val and Cys579Arg were not found to be reported before. We also observed four polymorphisms in patients, of which NM_000435.2:c.680+60A>G in intron 4 was found to be novel.

\section{Material and Methods}

\section{1. Patients:}

300 patients were tested for CADASIL by screening for mutations and polymorphisms in an exon screening procedure involving exons 3 and 4 initially, followed by screening of exons 2, 11, 18 and 19. Patients were referred to our laboratory through neurologists with samples from Australia and New Zealand for diagnostic testing.

\subsection{Molecular Analysis:}

\subsubsection{Samples:}

Sample format was either whole blood (in lithium heparin/EDTA tubes) or DNA. For whole blood samples, DNA was extracted using Qiagen QIAamp DNA Blood Midi Kits. Quantitation of all DNA samples was performed on Nanodrop ND1000.We used a positive control DNA sample which was selected such that its sequence did not have any polymorphisms or mutations for the exons screened. From the polymerase chain reaction (PCR) step to the sequencing step, each reaction was carried out in duplicate in order to get consistent results.

\subsection{2. $P C R:$}

The primers used for the PCR amplification of exons 2,3,4,11,18 and 19 are shown in Table 1. The protocol for the PCR amplification of exons 2, 4, 18 and 19 required the following reagents: Buffer F (Epicentre MasterAmp ${ }^{\mathrm{TM}}$ 2xPCR PreMix which contains $\mathrm{MgCl} 2$, dNTPs and MasterAmp ${ }^{\mathrm{TM}}$ PCR enhancers) -10ul, forward and reverse primers-0.5ul each (10uM), Taq polymerase (Promega GoTaq)-0.2ul, dH2O-7.8ul and 1ul of sample DNA (at 20ng/ul). For PCR of exon 3, we used the same protocol, except changing the buffer and using Buffer D (Epicentre MasterAmp ${ }^{\mathrm{TM}} 2 \mathrm{xPCR}$ PreMix) instead and the concentration of the primers were at 5uM. For PCR amplification of exon 11, we used 16.25ul of BufferF, 1.08ul each of forward and reverse primers (10uM), 0.26ul of Taq 
polymerase, $12.33 \mathrm{ul}$ of $\mathrm{dH} 2 \mathrm{O}$ and $1.5 \mathrm{ul}$ of sample DNA(at 20ng/ul). Cycling protocol for exons 2,3,11,18 and 19: $\left(94^{\circ} \mathrm{C}-4 \mathrm{~min}\right)-1$ cycle, $\left(94^{\circ} \mathrm{C}-1 \mathrm{~min}, 60^{\circ} \mathrm{C}-1 \mathrm{~min}\right)-35$ cycles, $\left(72^{\circ} \mathrm{C}-2 \mathrm{~min}\right.$ and $\left.4^{\circ} \mathrm{C}-2 \mathrm{~min}\right)-1$ cycle. Cycling protocol for exon 4 : $\left(94^{\circ} \mathrm{C}-4 \mathrm{~min}\right)-1$ cycle, $\left(94^{\circ} \mathrm{C}-1 \mathrm{~min}, 55^{\circ} \mathrm{C}-\right.$ $\left.1 \mathrm{~min}, 72^{\circ} \mathrm{C}-30 \mathrm{sec}\right)-35$ cycles, $\left(72^{\circ} \mathrm{C}-2 \min , 4^{\circ} \mathrm{C}-2 \mathrm{~min}\right)-1$ cycle.

\subsubsection{Sequencing:}

We purified the PCR products using Affymetrix ExoSAP-IT reagent which removes primers and additional dNTPs that were not used up in the PCR reaction. 5ul of PCR product was added to $1 \mathrm{ul}$ of ExoSAP-IT reagent and 4ul of distilled water was added to make a total of 10ul reaction. The cycling conditions for this step were: $\left(37^{\circ} \mathrm{C}-15 \mathrm{~min}\right)-1$ cycle, $\left(80^{\circ} \mathrm{C}-15 \mathrm{~min}\right)-1$ cycle and $\left(4^{\circ} \mathrm{C}-2 \mathrm{~min}\right)-$ 1cycle. The subsequent step was the labelling reaction carried out using ABI BigDye ${ }^{\mathrm{TM}}$ Terminator (BDT) v3.1 Cycle Sequencing chemistry. In this step, each sample was sequenced in both directions and the following reagents were used for the BDT step: 1.3ul of primer (forward/reverse), $3 \mathrm{ul}$ of $5 \mathrm{x}$ sequencing buffer, 5ul of BDT v3.1 reagent and 2ul of ExoSAP product (required 20ng/ul product which was calculated for each sample after the ExoSAP step) and 8.7ul of dH2O to make a total reaction volume of 20ul. Cycling protocol for BDT reaction: $\left(96^{\circ} \mathrm{C}-1 \mathrm{~min}\right)-1 \mathrm{cycle},\left(96^{\circ} \mathrm{C}-10 \mathrm{sec}\right.$, $\left.50^{\circ} \mathrm{C}-5 \mathrm{sec}, 60^{\circ} \mathrm{C}-4 \mathrm{~min}\right)-30$ cycles, $\left(4^{\circ} \mathrm{C}-5 \mathrm{~min}, 10^{\circ} \mathrm{C}-5 \mathrm{~min}\right.$ and $\left.4^{\circ} \mathrm{C}-2 \mathrm{~min}\right)-1$ cycle. Following this, the samples were purified and concentrated using ethanol precipitation with sodium acetate, EDTA and ethanol. We performed sequencing on an ABI 3130 Genetic Analyser.

\subsubsection{Analysis:}

Sequencing data was analysed with Chromas 2.33 software and compared with NCBI reference sequences. All sample sequences were also compared with the positive control sequences for each exon to ensure validity of the results.

\section{Results}

300 patients were tested for CADASIL, from which 65 patients $(21.7 \%)$ showed variations in the NOTCH3 gene. 37 of these patients (12.3\%) had mutations in the NOTCH3 gene and 32 patients $(10.7 \%)$ had non amino acid changing polymorphisms. Out of these 65 patients, four patients had both-a mutation and a polymorphism, one patient had three polymorphisms, one of which was an amino acid changing polymorphism (His170Arg) in the NOTCH3 gene while another patient had a known exonic polymorphism (Thr101Thr, rs3815188) in exon 3 along with an unreported intronic polymorphism NM_000435.2:c.680+60A>G in intron 4 of the NOTCH3 gene. The sequence results of the intronic polymorphism observed in a 46 year-old male is shown in Figure 1E. The majority of the 300 patients were screened for exons 3 and 4 and when no variations were observed, extended CADASIL test (exons 2, 11, 18 and 19) was offered, with 17 patients investigated for the extended testing. Figure 2 plots the number of mutations found in the respective exons with the number of patients and Table 2 gives a list of polymorphisms observed in CADASIL patients. Ala202Val 
observed in a 49 year-old female and Cys579Arg observed in a 66 year-old female were found to be two novel non-synonymous mutations in the NOTCH3 gene that have not been reported before. Ala202Val had a heterozygous variation at nucleotide position 590(C/T) and is shown in Figure 1A. The majority of the mutations in Figure 2 were found to be in exon 4 (nearly half the percentage) including the novel Ala202Val. Also, the majority of the mutations in Figure 2 indicate either a change in an arginine or a cysteine amino acid, including Cys579Arg which had a heterozygous variation at nucleotide position 1735 (T/C as shown in Figure 1C). The most common mutation found in 13 patients was located in exon 4 with a change from an arginine to cysteine at amino acid position 141 in the NOTCH3 gene. Thr101Thr in exon 3 was the most commonly occurring polymorphism, found in 19 patients. One patient had a His170Arg amino acid changing polymorphism and an Ala202Ala (rs1043994) polymorphism in exon 4, along with a Thr101Thr polymorphism in exon 3 of the NOTCH3 gene. The second most commonly occurring polymorphism, Ala202Ala located in exon 4, occurs at nucleotide position 606 in the NOTCH3 gene where there a change from nucleotide ' $G$ ' to 'A' but this causes no change in the amino acid at position 202. The Ala202Val mutation that we found also occurs at the same codon, at nucleotide position 605 in the NOTCH3 gene, but in this case, causes an amino acid change. We also observed a mother and daughter pair with the Arg141Cys mutation in exon 4 and two sisters with the Arg153Cys mutation in exon 4. One of the sisters had a son who also showed the same Arg153Cys mutation, along with a Thr101Thr polymorphism in the NOTCH3 gene.

\section{Discussion}

The principal cause for pathogenesis of CADASIL is still unclear. A bioinformatics study in 2003 [15] examined NOTCH3 genomic sequences for conservation locations across species. The outcome of their analysis suggested that deficiency in Notch3 signaling was not very probable in causing the phenotype. They proposed that the mutations in NOTCH3 are gain-of-function mutations as they occur in a nonconserved area and that protein deposits occurring due to misfolding and aggregation could be the likely cause of the phenotype. We did not observe any mutation in exon 2 of the NOTCH3 gene for any of our patients tested for CADASIL. The largest portion of the mutations were in exon 4 with six different types of mutations located in it as shown in in Figure 2. The most commonly occuring mutation in exon 4 was Arg141Cys observed in thirteen patients, then both Arg182Cys and Arg153Cys were observed in seven patients and our first novel mutation, Ala202Val also lies in this exon. Exon 11 had three different types of mutations including our second novel mutation Cys579Arg, each occuring only once. His170Arg (which causes an adenine to guanine nucleotide change at position 587 in exon 4) is an amino acid changing polymorphism[13] which we found in one of our patients who also had two other non amino acid changing polymorphisms, namely Ala202Ala (exon 4) and Thr101Thr (exon 3) in the NOTCH3 gene. But there is some discrepancy over the categorization of His170Arg. In 1997, Joutel et al. [13] described His170Arg as a non amino 
acid changing polymorphism. But in 2009, Ampuero et al. [16] conducted a study on Spanish subjects who were clinically suspected to have CADASIL and they described His170Arg as a mutation. Ala1020Pro (rs35769976) is another amino acid changing polymorphism which lies in exon 19 and has a nucleotide change from guanine to cytosine at position 3058 of the NOTCH3 gene [17]. However, there is again some controversy over the classification of Ala1020Pro as a mutation or a polymorphism. In 2008, Scheid et al. [18] reported this as a mutation and gave several reasons to justify their classification based on the reasoning that this variant was not observed in more than 100 controls, this variant affected the highly conserved portion in the EGF-like repeat domain 26 and that an amino acid change could modify secondary and tertiary structures causing effects on protein interactions.

Mutations in the NOTCH3 gene that are related to CADASIL are located in the EGF-like repeats. Most of these mutations either add or reduce a cysteine, leading to an odd number of cysteines in one of the 34 EGF-like repeats which is situated in the extracellular domain of the Notch3 protein [15]. Irregular number of cysteines in turn causes atypical disulphide bridging with another Notch3 molecule or another protein and eventually the three dimensional structure of Notch3 is altered [17]. Generally each EGF-repeat consists of six cysteine residues that form three disulphide bonds. When there are an odd number of cysteine residues, the disulphide bonds get disrupted and may cause conformational change of the domain. The free cysteine may be inclined towards oligomerization on the cell surface which could hinder Notch trafficking as is supported by the finding that there is Notch3 protein build up in CADASIL arteries. Another interesting finding indicates that most of the CADASIL mutations occur near the N-terminus of the Notch protein (function of this terminal is mostly unknown) and far from the putative ligand binding region (EGF 10-11) [19].

From literature, we found 135 known NOTCH3 variations in exons 2,3,4,11,18 and 19. Most of these variations involved a change in a cysteine molecule and occurred most frequently in exon 4 , followed by exon 3, then exon 2 and exon 11, both comprising similar numbers. The mutations that we observed for the two sisters, Arg153Cys (which was also observed in the son of one of the sisters) and also the mutation observed in the mother and daughter, Arg141Cys, involved a change in a cysteine molecule. In addition, we detected a polymorphism NM_000435.2:c.680+60A>G in intron 4 of the NOTCH3 gene which lies near the intron-exon boundary region and may have an effect on the splicing mechanism.

A study in 2004 by Peters [20] and group indicated that the EGF repeat domains of Notch receptors were essential for ligand interactions [20,21] but it is still unknown as to which EGF repeats of Notch3 are actually utilized in ligand binding. They also suggested some ways of looking at the pathogenic effects of mutations namely loss of receptor function, gain of receptor function and neomorphic (e.g. toxic) effects. Studies were conducted with R133C and C183R mutations located in exon 4 and they presented experimental data showing that loss or gain of Notch3 receptor function was not the primary mechanism in CADASIL as both of these mutations were associated with normal 
signaling activity. Nevertheless they indicated that limitations of their study were that their work was restricted to the major Notch signaling pathway involving Delta, Jagged ligands and RBP-Jk and indicated that CADASIL mutations may affect other Notch signaling pathways. They also indicated that small oligomers of misfolded proteins may be toxic to cells as proposed by various studies on adult-onset neurological conditions [20,22].

As mentioned above in section 4, protein deposits due to aggregation may be one of the main causes leading to the CADASIL phenotype. Peters et al. [20] also suggested that a major reason for aggregation to occur was when the ligand bound to the mutant Notch3 receptor as it then released the extracellular domain that accumulates in CADASIL patients. However, the mechanisms of Notch3 receptor aggregation remain unknown. Another theory suggested that the cause for CADASIL pathology may be due to the indirect effect of irregular Notch3 protein accumulation or as a direct effect of the disturbance of the Notch signal regulation or a combination of both [19]. Further studies are required to determine the role of detected variants in CADASIL pathogenesis.

\section{Conclusion}

We tested 300 patients for CADASIL and 65 patients (21.7\%) showed variations in the NOTCH3 gene. Out of these, 37 (12.3\%) had mutations in the NOTCH3 gene and 32 (10.7\%) had non amino acid changing polymorphisms. We report two novel non-synonymous mutations in exon 4 and exon 11 of the NOTCH3 gene. The first mutation in exon 4 was an alanine to valine amino acid substitution at position 202 which is also the common codon site for the second most frequently occurring polymorphism, Ala202Ala, in our patient cohort. The second novel mutation in exon 11 was a cysteine to arginine amino acid substitution at position 579 . This mutation can be categorized into the most ubiquitous type, cysteine changing mutations found in the NOTCH3 gene. All the CADASIL related mutations in the NOTCH3 gene are situated in the EGF-like repeats and irregular number of cysteines will affect the disulphide bridging and eventually may modify the three dimensional structure of the Notch3 protein. We also report a polymorphism, NM_000435.2:c.680+60A $>$ G in intron 4 which has not been described before. We do not yet know the characteristics of these novel variations and further investigation is required at the transcriptomics and proteomics level.

\section{Conflict of Interest}

The authors declare that there are no conflicts of interest.

\section{Acknowledgements}

We wish to thank all the members of Genomics Research Centre lab and the clinic for their support. 


\section{References:}

[1] P. Sourander, J. Walinder, Hereditary multi-infarct dementia, Acta Neuropathologica, 39 (1977) 247-254.

[2] W. Robinson, S.L. Galetta, L. McCluskey, M.S. Forman, L.J. Balcer, Retinal findings in cerebral autosomal dominant arteriopathy with subcortical infarcts and leukoencephalopathy (cadasil), Surv Ophthalmol, 45 (2001) 445-448.

[3] E. Tournier-Lasserve, A. Joutel, J. Melki, J. Weissenbach, G.M. Lathrop, H. Chabriat, J.L. Mas, E.A. Cabanis, M. Baudrimont, J. Maciazek, M.A. Bach, M.G. Bousser, Cerebral autosomal dominant arteriopathy with subcortical infarcts and leukoencephalopathy maps to chromosome 19q12, Nat Genet, 3 (1993) 256-259.

[4] M.M. Ruchoux, Cerebral autosomal dominant arteriopathy with subcortical infarcts and leukoencephalopathy: A genetic form of vascular dementia, 4 (2004) 102-108.

[5] H. Chabriat, K. Vahedi, M.G. Bousser, M.T. Iba-Zizen, A. Joutel, A. Nibbio, T.G. Nagy, E. Tournier-Lasserve, M.O. Krebs, J. Julien, X. Ducrocq, M. Levasseur, J.L. Mas, B. Dubois, P. Homeyer, O. Lyon-Caen, Clinical spectrum of CADASIL: a study of 7 families, Lancet, 346 (1995) 934-939.

[6] M.M. Ruchoux, D. Guerouaou, B. Vandenhaute, J.P. Pruvo, P. Vermersch, D. Leys, Systemic vascular smooth muscle cell impairment in cerebral autosomal dominant arteriopathy with subcortical infarcts and leukoencephalopathy, Acta Neuropathologica, 89 (1995) 500-512.

[7] A. Joutel, C. Corpechot, A. Ducros, K. Vahedi, H. Chabriat, P. Mouton, S. Alamowitch, V. Domenga, M. CÉCillion, E. MarÉChal, J. Maciazek, C. VayssiÈRe, C. Cruaud, E.A. Cabanis, M.M. Ruchoux, J. Weissenbach, J.F. Bach, M.G. Bousser, E. Tournier-Lasserve, Notch3 Mutations in Cerebral Autosomal Dominant Arteriopathy with Subcortical Infarcts and Leukoencephalopathy (CADASIL), a Mendelian Condition Causing Stroke and Vascular Dementia, Annals of the New York Academy of Sciences, 826 (1997) 213-217.

[8] A. Joutel, C. Corpechot, A. Ducros, K. Vahedi, H. Chabriat, P. Mouton, S. Alamowitch, V. Domenga, M. Cecillion, E. Marechal, J. Maciazek, C. Vayssiere, C. Cruaud, E.A. Cabanis, M.M. Ruchoux, J. Weissenbach, J.F. Bach, M.G. Bousser, E. Tournier-Lasserve, Notch3 mutations in CADASIL, a hereditary adult-onset condition causing stroke and dementia, Nature, 383 (1996) 707 710.

[9] S. Artavanis-Tsakonas, K. Matsuno, M. Fortini, Notch signaling, Science, 268 (1995) 225-232. [10] Y.C. Lee, A.H. Yang, H.C. Liu, W.J. Wong, Y.C. Lu, M.H. Chang, B.W. Soong, Cerebral autosomal dominant arteriopathy with subcortical infarcts and leukoencephalopathy: Two novel mutations in the NOTCH3 gene in Chinese, Journal of the Neurological Sciences, 246 (2006) 111115. 
[11] A. Joutel, F. Andreux, S. Gaulis, V. Domenga, M. Cecillon, N. Battail, N. Piga, F. Chapon, C. Godfrain, E. Tournier-Lasserve, The ectodomain of the Notch3 receptor accumulates within the cerebrovasculature of CADASIL patients, J Clin Invest, 105 (2000) 597-605.

[12] A. Joutel, D.D. Dodick, J.E. Parisi, M. Cecillon, E. Tournier-Lasserve, M.G. Bousser, De novo mutation in the Notch3 gene causing CADASIL, Ann Neurol, 47 (2000) 388-391.

[13] A. Joutel, K. Vahedi, C. Corpechot, A. Troesch, H. Chabriat, C. Vayssiere, C. Cruaud, J. Maciazek, J. Weissenbach, M.G. Bousser, J.F. Bach, E. Tournier-Lasserve, Strong clustering and stereotyped nature of Notch3 mutations in CADASIL patients, Lancet, 350 (1997) 1511-1515.

[14] L. Huang, Q. Yang, L. Zhang, X. Chen, Q. Huang, H. Wang, Acetazolamide improves cerebral hemodynamics in CADASIL, J Neurol Sci, 292 (2010) 77-80.

[15] C.P. Donahue, K.S. Kosik, Distribution pattern of Notch3 mutations suggests a gain-of-function mechanism for CADASIL, Genomics, 83 (2004) 59-65.

[16] I. Ampuero, J. Alegre-Abarrategui, I. Rodal, A. Espana, R. Ros, J.L. Sendon, E.G. Galloway, A. Cervello, A.B. Caminero, A. Zabala, E. Erro, F. Jarauta, L. Morlan, E. Lopez-Valdes, Y. Aladro, M. Seijo, G.G. Rivas, D.G. Munoz, J.G. de Yebenes, On the diagnosis of CADASIL, J Alzheimers Dis, 17 (2009) 787-794.

[17] C. Ungaro, R. Mazzei, F.L. Conforti, T. Sprovieri, P. Servillo, M. Liguori, L. Citrigno, A.L. Gabriele, A. Magariello, A. Patitucci, M. Muglia, A. Quattrone, CADASIL: extended polymorphisms and mutational analysis of the NOTCH3 gene, J Neurosci Res, 87 (2009) 1162-1167.

[18] R. Scheid, W. Heinritz, T. Leyhe, D.R. Thal, R. Schober, S. Strenge, D.Y. von Cramon, U.G. Froster, Cysteine-sparing notch3 mutations: cadasil or cadasil variants?, Neurology, 71 (2008) 774776.

[19] T. Wang, M. Baron, D. Trump, An overview of Notch3 function in vascular smooth muscle cells, Prog Biophys Mol Biol, 96 (2008) 499-509.

[20] N. Peters, C. Opherk, S. Zacherle, A. Capell, P. Gempel, M. Dichgans, CADASIL-associated Notch3 mutations have differential effects both on ligand binding and ligand-induced Notch3 receptor signaling through RBP-Jk, Exp Cell Res, 299 (2004) 454-464.

[21] I. Rebay, R.J. Fleming, R.G. Fehon, L. Cherbas, P. Cherbas, S. Artavanis-Tsakonas, Specific EGF repeats of Notch mediate interactions with Delta and Serrate: implications for Notch as a multifunctional receptor, Cell, 67 (1991) 687-699.

[22] M. Bucciantini, E. Giannoni, F. Chiti, F. Baroni, L. Formigli, J. Zurdo, N. Taddei, G. Ramponi, C.M. Dobson, M. Stefani, Inherent toxicity of aggregates implies a common mechanism for protein misfolding diseases, Nature, 416 (2002) 507-511. 
Tables and Figures:

\begin{tabular}{|c|c|}
\hline Exon\# & Primer Sequences (5' to 3') \\
\hline \multirow[t]{2}{*}{2} & Forward : GAGGGGGTTTGTCACTTGG \\
\hline & Reverse : ACACACAGGGCCCACTGGT \\
\hline \multirow[t]{2}{*}{3} & Forward : GCGTGTTTCTTGCCTGTCTTGTGT \\
\hline & Reverse : AGGACAGGGTGAGTTTAGGACTGA \\
\hline \multirow[t]{2}{*}{4} & Forward : TAGTCGGGGGTGTGGTCAGT \\
\hline & Reverse : TCAAACCCTAGCAGGGAA \\
\hline \multirow[t]{2}{*}{11} & Forward : ATTGGTCCGAGGCCTCACTT \\
\hline & Reverse : CCATTCCCAACCССТCTGTG \\
\hline \multirow[t]{2}{*}{18} & Forward : GGGGAAGCACTCAGAGTCAG \\
\hline & Reverse : AGGTCCCCAGTAACTCCA \\
\hline \multirow[t]{2}{*}{19} & Forward : CCAGGTGGGTGGAGTTACTGGGG \\
\hline & Reverse : AGCAGGAGGTACGTGCATGA \\
\hline
\end{tabular}

\begin{tabular}{|llll|}
\hline No. & Polymorphisms & Exon\# & Number of patients \\
1 & Thr101Thr & Exon 3 & 19 \\
2 & Ala202Ala & Exon 4 & 14 \\
3 & His170Arg & Exon 4 & 1 \\
4 & NM_000435.2:c.680+60A $>$ G (Novel) & Intron 4 & 1 \\
\hline
\end{tabular}


Sequence Analysis

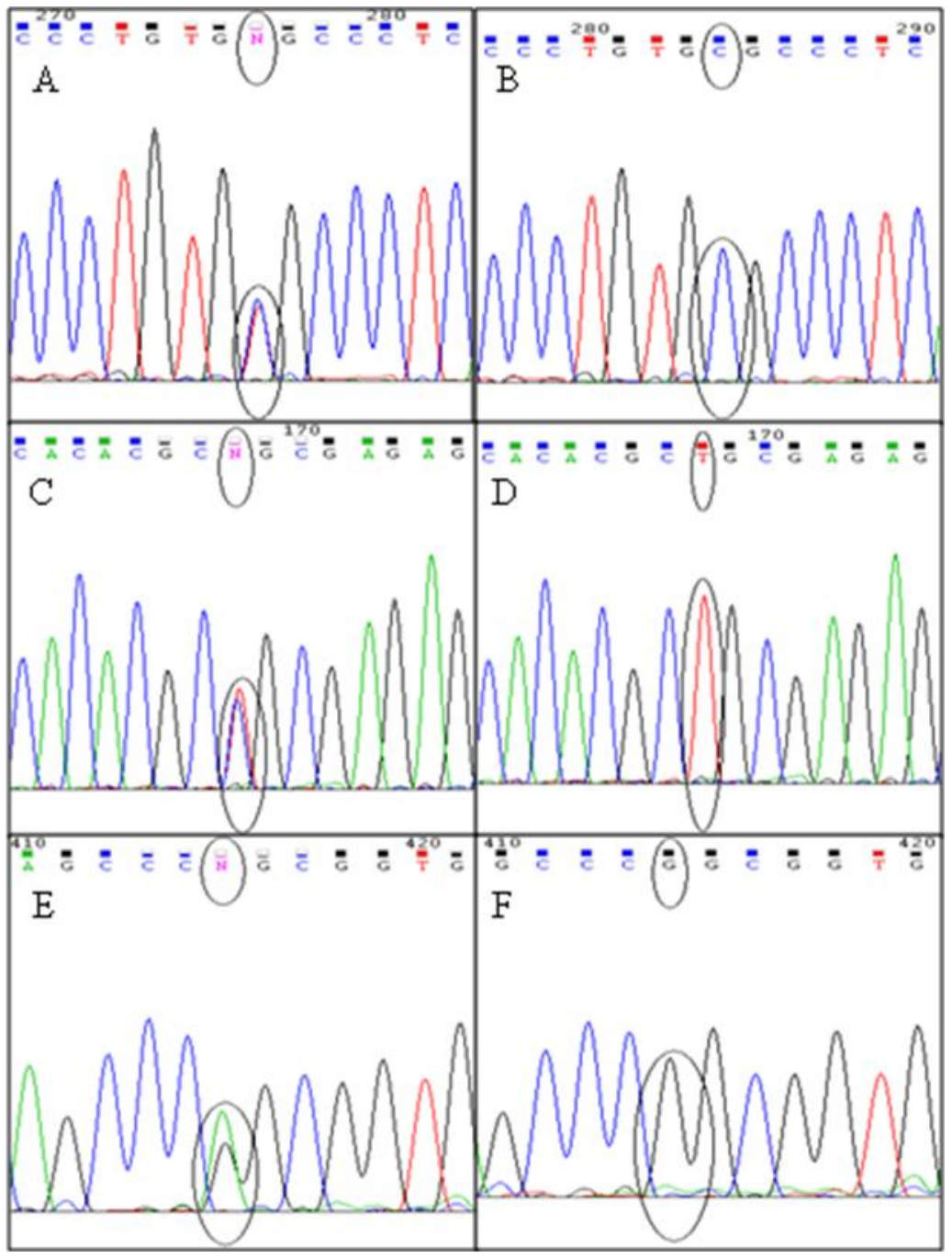

Fig1. Sequence traces for the novel variations are compared with the wildtype sequences.

(A): Ala202Val mutation in exon 4 (heterozygous C/T), (B): Wildtype (CC) in exon 4, (C):

Cys579Arg mutation in exon 11 (heterozygous T/C), (D): Wildtype (TT) in exon11, (E):

Heterozygous polymorphism NM_000435.2:c.680+60A>G in intron 4 and (F): Wildtype (GG) in intron 4. 
Mutations detected in our patient cohort

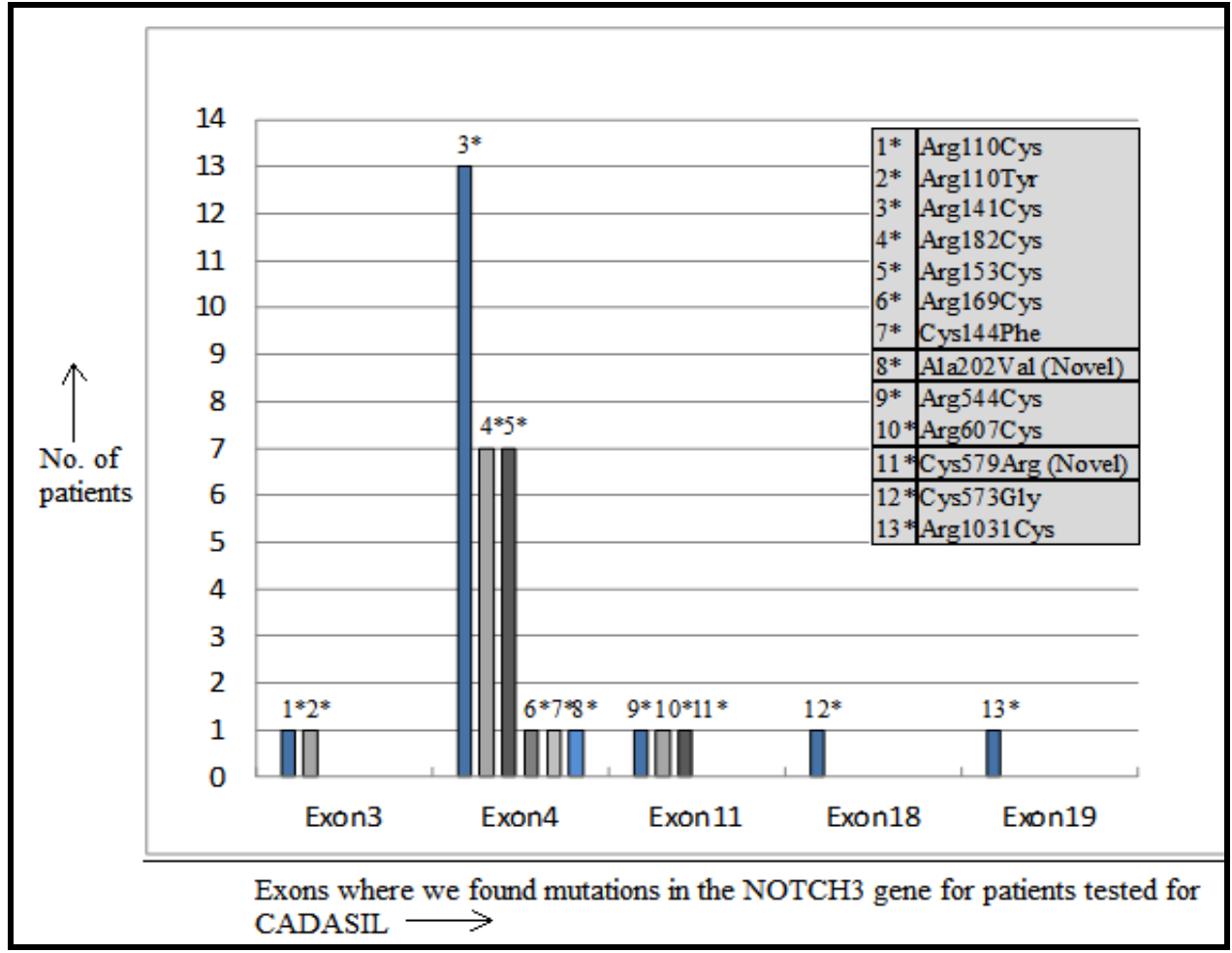

Fig2. This figure plots the number of mutations observed in 37 patients who were tested for CADASIL. 